\title{
2 Presence of sodium taurocholate co-transporting 3 polypeptide and Hepatitis $B$ replication marker on placenta: Another home for the virus
}

Garima Garg ${ }^{1}$, Meenu MN¹ , Kajal Patel ${ }^{1}$, Shashank Purwar ${ }^{1}$, Sramana Mukhopadhyay² ${ }^{2}$ Nitu Mishra ${ }^{3}$, Sudheer Gupta $^{1}$, Sumit Kumar Rawat ${ }^{4}$, Ritu Khosla ${ }^{5}$, Jitendra Singh ${ }^{6}$, Shashwati Nema ${ }^{1}$, Debasis Biswas ${ }^{1}$, Anirudh K Singh $^{1}$, Ashish Kumar Vyas ${ }^{1 *}$

${ }^{1}$ Department of Microbiology, All India Institute of Medical Sciences, Bhopal, INDIA

${ }^{2}$ Department of Pathology, All India Institute of Medical Sciences, Bhopal, INDIA

${ }^{3}$ Department of Gynecology \& Obstetrics, Gandhi Medical College, Bhopal, INDIA

${ }^{4}$ Department of Microbiology, Bundelkhand Medical College, Sagar, INDIA

${ }^{5}$ Department of Biomedical Science, University of Delhi, INDIA

${ }^{6}$ Department of Translational Medicine Centre, All India Institute of Medical Sciences, Bhopal, INDIA

\section{*Correspondence:}

Dr. Ashish Kumar Vyas, Ph.D.

Department of Microbiology,

All India Institute of Medical Sciences, Bhopal, INDIA

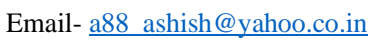

\begin{abstract}
Background: The role of sodium taurocholate co-transporting polypeptide (NTCP), in facilitating the binding of Hepatitis B virus (HBV) on surface of hepatocytes is well documented. Expression of NTCP in extra hepatic cells may make these cells susceptible to HBV infection and support cellular proliferation akin to hepatocytes. Placental replication of HBV is not well explored. In this study we have assessed the expression of NTCP and HBV replication markers ( $\mathrm{HBeAg}, \mathrm{HBcAg}$, and $\mathrm{HBV}$ DNA) in placental cells, to investigate if these cells act as host for HBV. Methods: Fourty one HBsAg+ve pregnant women along with 10 healthy controls were enrolled after obtaining informed consent. The HBV DNA in placenta was detected by qPCR using primers for X and core ORF. Expression of NTCP in placenta was analyzed by qRT-PCR and further investigated by immunohistochemistry (IHC) along with HBV replication biomarkers, HBeAg, and HBcAg. Results: HBsAg positive subjects were divided in two groups on the basis of viral load [High Viral Load (HVL) Group; viral load $\geq 2000 I U / m l$, Low Viral Load (LVL) Group; viral load <2000IU/ml] according to INASL guidelines 2018. HBV infected females showed increased expression of NTCP in trophoblasts of placenta compared to control group (HVL 3.69 \pm 0.13 Vs Control 1.74 \pm 0.15 , $\mathrm{p}=0.0117$ ). Furthermore, significant difference in NTCP expression was also observed between HVL and LVL group (HVL $3.69 \pm 0.13$ Vs LVL $1.98 \pm 0.17, \mathrm{p}=0.022$ ) and positively correlated with the maternal HBV DNA load. Membranous and/or cytoplasmic immunostaining of NTCP, and cytoplasmic staining of HBeAg and HBcAg in trophoblasts along with presence of HBV DNA indicated that trophoblasts are not only susceptible to HBV infection but may also be a site for viral replication. Conclusions: This is the pioneer study, which demonstrates expression of NTCP on placenta which may facilitate the entry of HBV. Furthermore, the study establishes the presence of $\mathrm{HBeAg}$ in placenta of patients without circulating $\mathrm{HBeAg}$, indicating
\end{abstract}




\section{Introduction} transmission.

\section{Results}

these cells may act as replication host/reservoir. This pioneering finding hints at the possibility of exploring the potential of NTCP blocking strategies in preventing vertical transmission of HBV.

Key words-Hepatitis B Virus, vertical transmission, Placenta, and Pregnancy, Sodium Taurocholate Co-transporting Polypeptide (NTCP).

Chronic Hepatitis B (CHB) infection is a major global health problem with 257 million infected people worldwide resulting in 887000 deaths annually [1 ]. Vertical transmission of HBV is a leading cause of chronicity ${ }^{2}$. About 90-95\% children acquiring infection from mother develop chronicity compared to adults where this risk is just 5$10 \%$. vertical transmission is main route of infection in developing countries. In India, 1-4\% of population is chronic carrier of HBV. Patients with CHB have high risk of developing end-stage cirrhosis and hepatocellular carcinoma[3]. Maternal hepatitis B e antigen ( $\mathrm{HBeAg})$ and high viral load are associated with $\mathrm{HBV}$ transmission to their newborns [2,4]. Earlier studies suggest that among the three mode of vertical transmission (Intrauterine or prenatal, Natal and Postnatal) [5]. However, precise mechanism of trans-placental routes of infection and vertical transmission of mother to baby are understudied. In one of the few available studies, we recently determined the role of maternal immunity in mother to baby vertical transmission[6] and the increased expression of HBV entry co-receptor Asialoglycoprotein receptor (ASGPR) in placental cells of transmitting mothers [7].

Sodium Taurocholate Co-transporting Polypeptide (NTCP) is a functional receptor of HBV. NTCP is an integral membrane glycoprotein involved in uptake of glycine/taurine-conjugated bile acids. It is considered to be exclusively expressed on hepatocytes [8]. Presence of NTCP is a key determinant of hepatotropism of HBV but its presence on placental cells is largely unknown and if present, it may play a crucial role in vertical transmission. Studies in primary trophoblast culture showed susceptibility of trophoblasts for HBV infection but there has been no such study on clinical specimens of placenta.

Previously serological studies showed that HBeAg can cross the placenta [9] but whether replication occurs in placenta was not explored. After getting entry through NTCP, does HBV also replicate inside trophoblasts is a considerable question.

In the present study, we not only found the expression of NTCP on placental trophoblasts, but also observed the presence of HBeAg and HBV DNA (both markers of active HBV replication) in trophoblast cells. Thus, through demonstration of both HBV receptor and replication in placental tissues, this study generates novel evidence for the placenta acting as a target and reservoir organ for this virus and provides a mechanistic basis for vertical

\subsection{Virological and serological characteristics}


The demographic and biochemical profile of subjects enrolled in the study is shown in Table 1. We did not find difference in base line clinical parameter between the HVL and LVL groups. Serum HBeAg analysis in HVL group showed $26.66 \%$ and none were positive in LVL group.

\subsection{Presence of $\mathrm{HBV}$ infection in placenta}

Presence of HBsAg and $\mathrm{HBcAg}$ in placenta denotes the infection of HBV in placental cells [9,11]. In present study we confirmed the HBV infection in placental cells by determining HBcAg by IHC. HBcAg was present in more than 70\% samples of HVL group and approximately 80\% samples of LVL group (Figure $1 \mathrm{~A}$ ). Control placentas were also examined for any nonspecific binding. We did not find any significant difference in the IHC score of HBcAg in HVL and LVL groups (HVL 94.62 \pm 9.46 Vs LVL 76.5 \pm 8.68 p = 0.9253) Figure 1 B).

\begin{tabular}{cccc}
\hline Parameter & Control $(\mathbf{n = 1 0})$ & LVL $(\mathbf{n}=\mathbf{1 9})$ & HVL(n=22) \\
\hline Age years & $23.9 \pm 3.755$ & $22.47 \pm 2.54$ & $24.51 \pm 3.17$ \\
\hline HBV DNA & -- & 39.2 & $3.0^{*} 10^{6}$ \\
$(\mathbf{I U} / \mathbf{m l})$ & & $(3.58-1790)$ & $\left(2.2^{*} 12^{3}-4.78 \times 10^{8}\right)$ \\
\hline AST $(\mathbf{I U})$ & $19.9 \pm 15.75$ & $99.81 \pm 67.57$ & $105.6 \pm 95.85$ \\
\hline ALT $(\mathbf{I U})$ & $29.51 \pm 12.24$ & $78.38 \pm 37.48$ & $56.71 \pm 34.28$ \\
\hline HBeAg & -- & $\mathrm{NIL}$ & $26.66 \%$ \\
\hline
\end{tabular}

Fig 1 Confirmation of Hepatitis B Virus infection in placenta

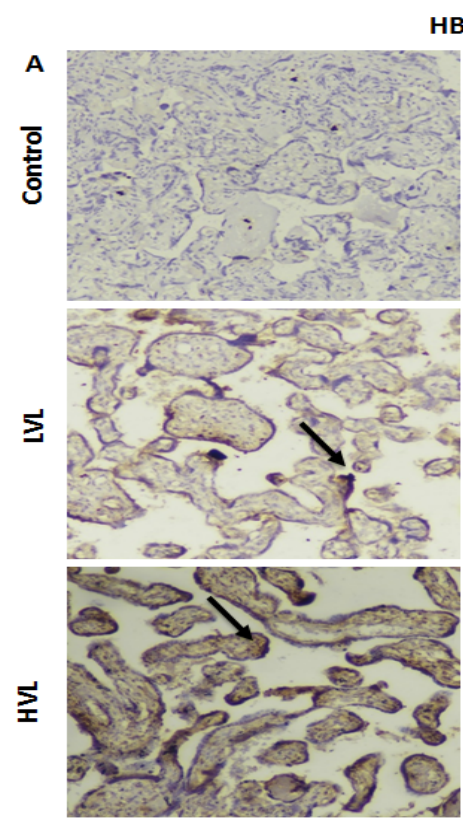

$100 x$

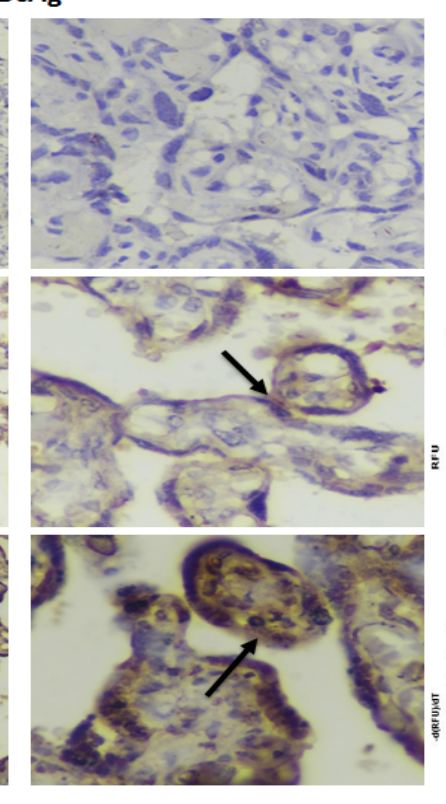

$400 x$

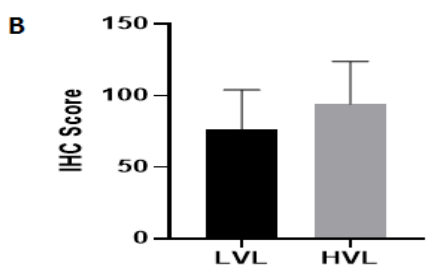

C Total HBV DNA
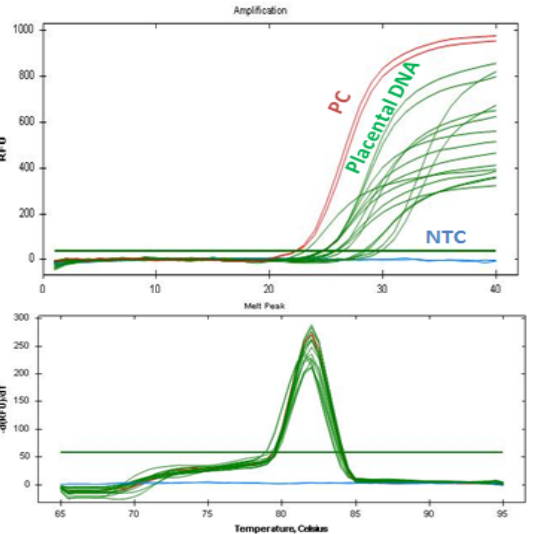

Figure 1. Representative Figure showed the presence of $\mathrm{HBcAg}$ in placenta through Immunohistochemistry (IHC), cytoplasmic staining in LVL and HVL groups was observed, and no staining in control (A). IHC Scores 
of HVL and LVL groups showed no significant difference (HVL 71 \pm 7.22 Vs LVL 65.5 $\pm 7.43 p=0.8687$ ). Significance was considered $p$-value was calculated for a risk threshold $\alpha=0.05, p^{*}<0.0332$ (B). Detection of total HBV DNA in placenta through qPCR Amplification curve and single peak in melt curve (C). HVL- High Viral Load Group, LVL- Low Viral Load Group, PC- Positive Control, NTC- No Template Control As presence of HBV DNA is also considered as a marker of infection, we performed qPCR for $\mathrm{X}$ and Core ORF to detect HBV DNA was detected in placental samples (supplementary Figure 1) from both groups and found that $92.30 \%$ of $\mathrm{HVL}$ and $62.5 \%$ of LVL samples gave positive amplification for these targets (Figure $1 \mathrm{C}$ ). The high percentage of HBV DNA positivity in both groups and stringent positivity analysis through $\mathrm{HBcAg}$ indicated placental infection in most of the samples. Our next aim wais to investigate whether HBV entry receptor NTCP expresses on placental cells.

\subsection{NTCP expresses on placental cells}

Encouraged by finding markers for HBV infection in placenta, we postulated that placenta may act as reservoir and facilitate viral replication. NTCP is the primary receptor for virus on hepatocytes and is thought to be exclusive to the cells. However, for placental cells to act as reservoir, these cells must express NTCP receptor. We started with probing the expression of receptor in placenta. The expression of NTCP was searched in the data submitted for different cell types/tissues to the "The Human Protein Atlas" (www.proteinatlas.org). The consensus normalized expression for single cell RNA (nTPM) from all single cell types have been shown in Figure 2.

Fig 2 Plot showing the consensus normalized expression of NTCP in different cell types

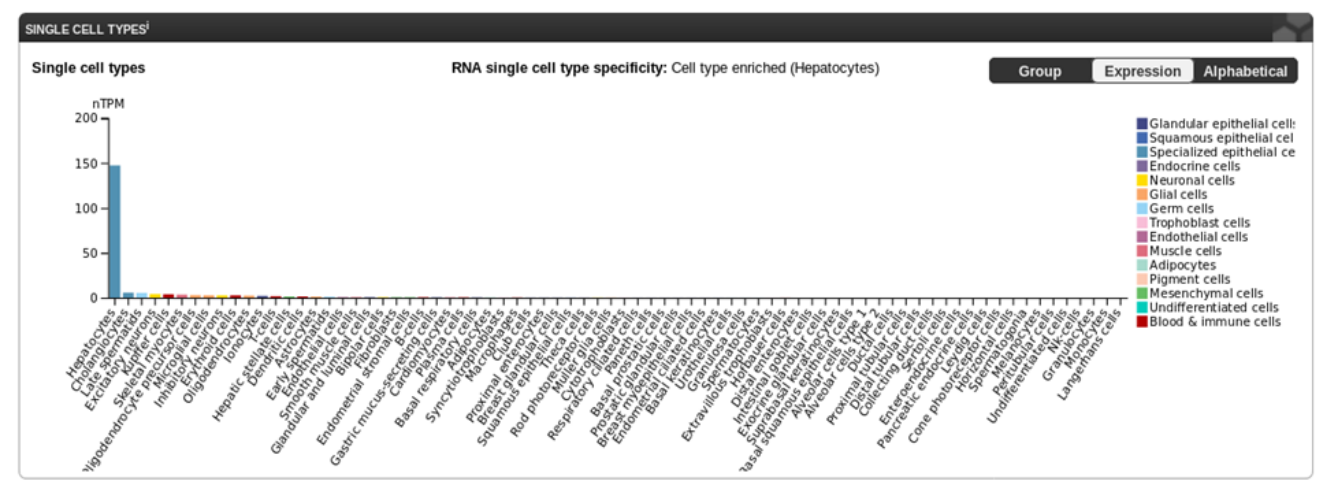

Figure 2. Color-coding in the plot, is based on cell type groups, each consisting of cell types with functional features in common.

It can be observed that the expression of NTCP in healthy human cell types is reported mainly in Hepatocytes and to a lower level in Cholangiocytes, Late spermatids, Excitatory neurons, Kupffer cells, Skeletal myocytes, Oligodendrocyte precursor cells, Microglial cells, Erythroid cells, Inhibitory neurons, Oligodendrocytes, Ionocytes, T-cells, Dendritic cells, Hepatic stellate cells, Astrocytes, Early spermatids, Endothelial cells, Smooth muscle cells \& B-cells. The cells specific to placenta have not been reported to express NTCP in the studies available in "The Human Protein Atlas".

We started examining expression of NTCP in our placental samples and interestingly found that NTCP is expressed in placenta as well (Figure 3). Quantification of NTCP expression was done by qRT-PCR. While 
NTCP expression in LVL group was comparable to the control group (LVL 1.98 \pm 0.17 Vs Control 1.74 \pm 0.15 , $\mathrm{p}=0.9213 ; *<0.0332$ ), it was significantly upregulated in HVL group when compared with control group (HVL 3.69 \pm 0.13 Vs Control 1.74 $\pm 0.15, \mathrm{p}=0.0117$; $* 0.0332$ ) or LVL group (HVL 3.69 \pm 0.13 Vs LVL 1.98 \pm 0.17 , $\mathrm{p}=0.022 ; *<0.0332$ ). This suggest that NTCP expression might be associated with viral load (Figure 3 A). To further ensure NTCP is translated we performed Immunohistochemistry IHC using NTCP specific antibody. Membranous and / or cytoplasmic staining was considered positive with reference to liver as positive control. Expression of NTCP was observed mainly in trophoblasts while stromal and endothelial cells were not stained (Figure 3 B). Membranous expression was largely observed in HVL group placenta. For confirmation of specificity of NTCP antibodies we also analyzed the IHC of other tissues (Breast tissue, Myometrium and Endometrium of uterus) for NTCP expression. (Supplementary Figure 2). As expected, we did not find expression of NTCP on breast tissue and layers of uterus, circumvents postnatal and prenatal risk, respectively. There was a significant difference between HVL and control group (HVL 185.5 \pm 9.71 Vs Control 65.5 $\pm 5.4, \mathrm{p}=$ 0.011; *<0.0332). Whereas, no significant difference was obtained between HVL and LVL groups (HVL

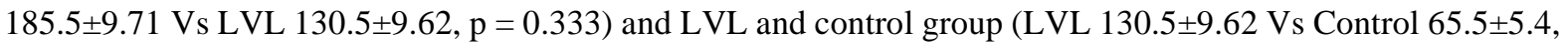
$\mathrm{p}=0.221)($ Figure $3 \mathrm{C})$.
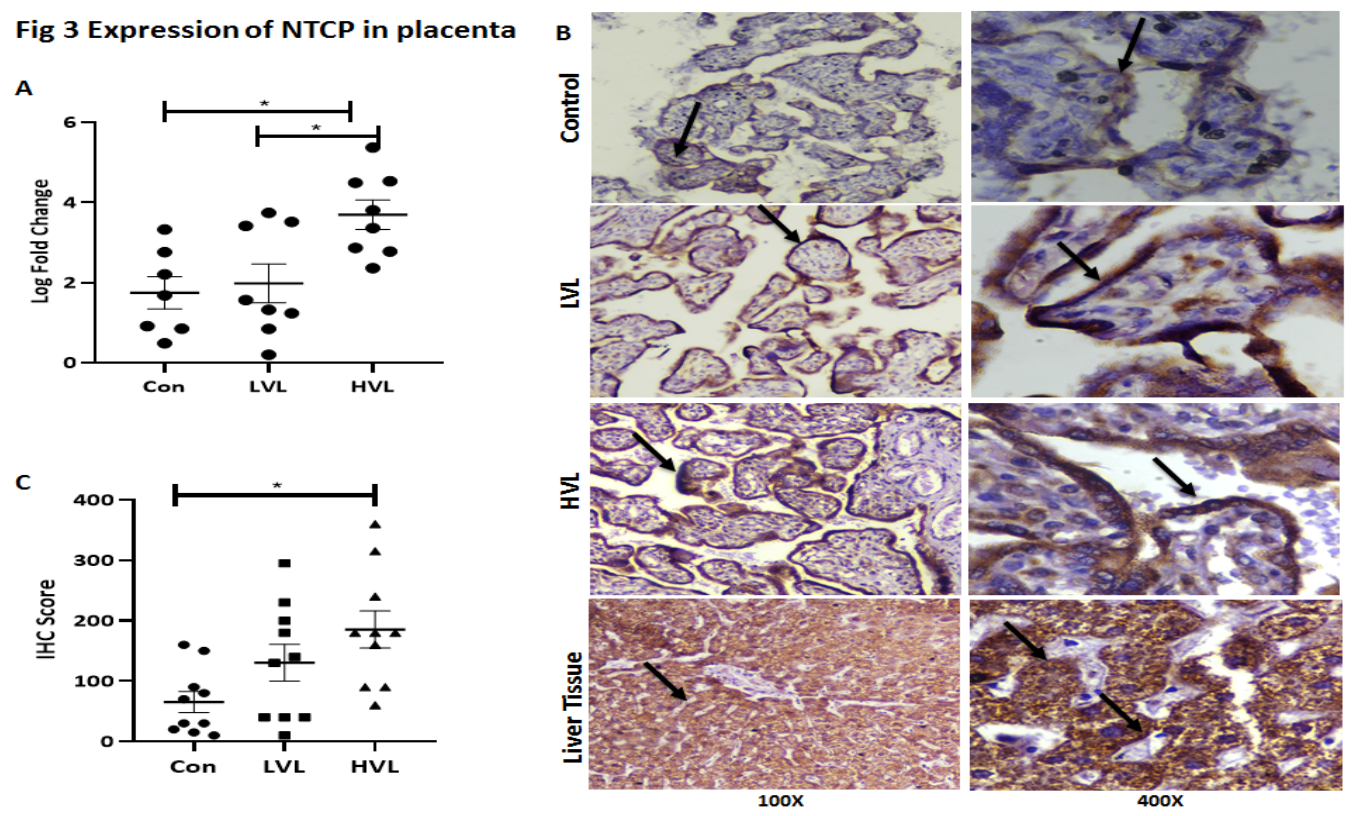

132

133

134

135

136

137

138

139

140

Figure 3. The log fold change in expression of NTCP in placenta among the groups (A). Representative Figure of expression of NTCP in placenta through Immunohistochemistry, weak cytoplasmic staining in control, moderate to strong membranous and cytoplasmic staining in LVL and HVL group was observed. Strong membranous and cytoplasmic staining of non pathological liver tissue was served as positive control (B). IHC Scores were plotted against log of HBV DNA viral load (IU/ml) (C).

Con- Control, HVL- High Viral Load Group, LVL- Low Viral Load Group

Presence of NTCP on placental cells indicate another home for HBV to validate this point we have assess the markers of HBV replication on placental cells. 

viral replication, in trophoblasts using IHC. Interestingly, we found the presence of $\mathrm{HBeAg}$ in trophoblast cells. We observed cytoplasmic staining in the $40 \%$ HVL group and $80 \%$ LVL group while there was no staining in the control group placentas showing specific immunostaining (Figure. $4 \mathrm{~A}$ ).

Fig 4 Presence of $\mathrm{HBeAg}$ in placenta

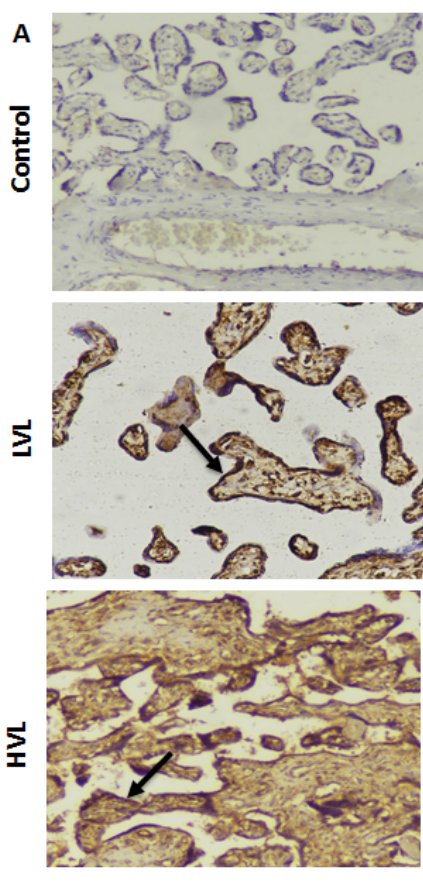

$100 \mathrm{X}$

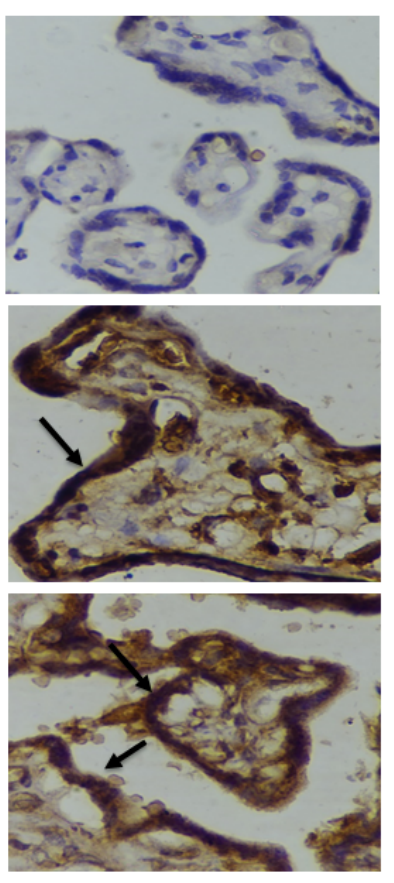

$400 \mathrm{X}$

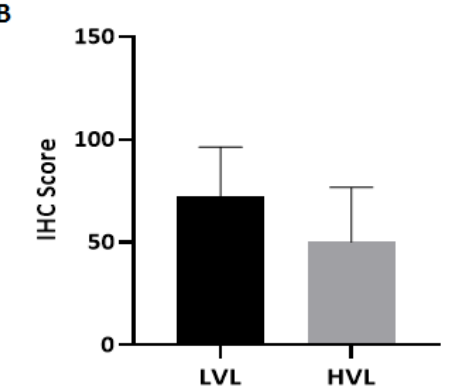

Figure 4 Representative Figure of detection of HBeAg in placenta through Immunohistochemistry, no staining in control and strong cytoplasmic staining in LVL and HVL groups was observed (A). IHC Scores of HVL and LVL between the groups (B). Two-tailed p-value was calculated for a risk threshold $\alpha=0.05 . p^{*}<0.0332$.

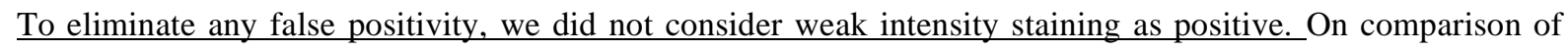
percentage of samples positive for peripheral and/or placental HBeAg, we found only $7.14 \%$ was positive for both while $14.28 \%$ were positive for HBeAg in circulation but not for placenta showing not all the peripheral positivity turned to placental. Surprisingly in $50 \%$ cases we found placental positivity but not peripheral affirming our finding that $\mathrm{HBeAg}$ is placenta derived as a result of active replication and not infiltered from periphery and $28.57 \%$ was negative for both. We did not find significant difference between the presence of HBeAg in HVL and LVL groups (HVL 50 \pm 8.43 Vs LVL $72.5 \pm 7.51 \mathrm{p}=0.2204 ; *<0.0332$ ) (Figure 4 B). Our finding strongly indicates that $\mathrm{HBV}$ can also replicate in placenta.

\subsection{Association of peripheral viral load with placental HBV infection markers and NTCP}

In order to analyse the correlation between placental infection markers (i.e., $\mathrm{HBcAg}$ and $\mathrm{HBeAg}$ ) and NTCP with viral load, we found a significant positive correlation between log fold change of NTCP expression with viral load $\left(\mathrm{PC}=0.7118, \mathrm{R}^{2}=0.3796, \mathrm{p}=0.0027^{* *} ; \mathrm{p}^{* *} 0.0021\right)$ although correlation was not significant when we compared IHC scores of NTCP $\left(\mathrm{PC}=0.3577, \mathrm{R}^{2}=0.0518\right)$ indicating post transcriptional modulation which has to be assessed on larger sample size. We also did correlation analysis with peripheral viral load with IHC 

correlation was found.

Fig 5 Correlation of Peripheral viral load with IHC score of NTCP, HBcAg and HBeAg
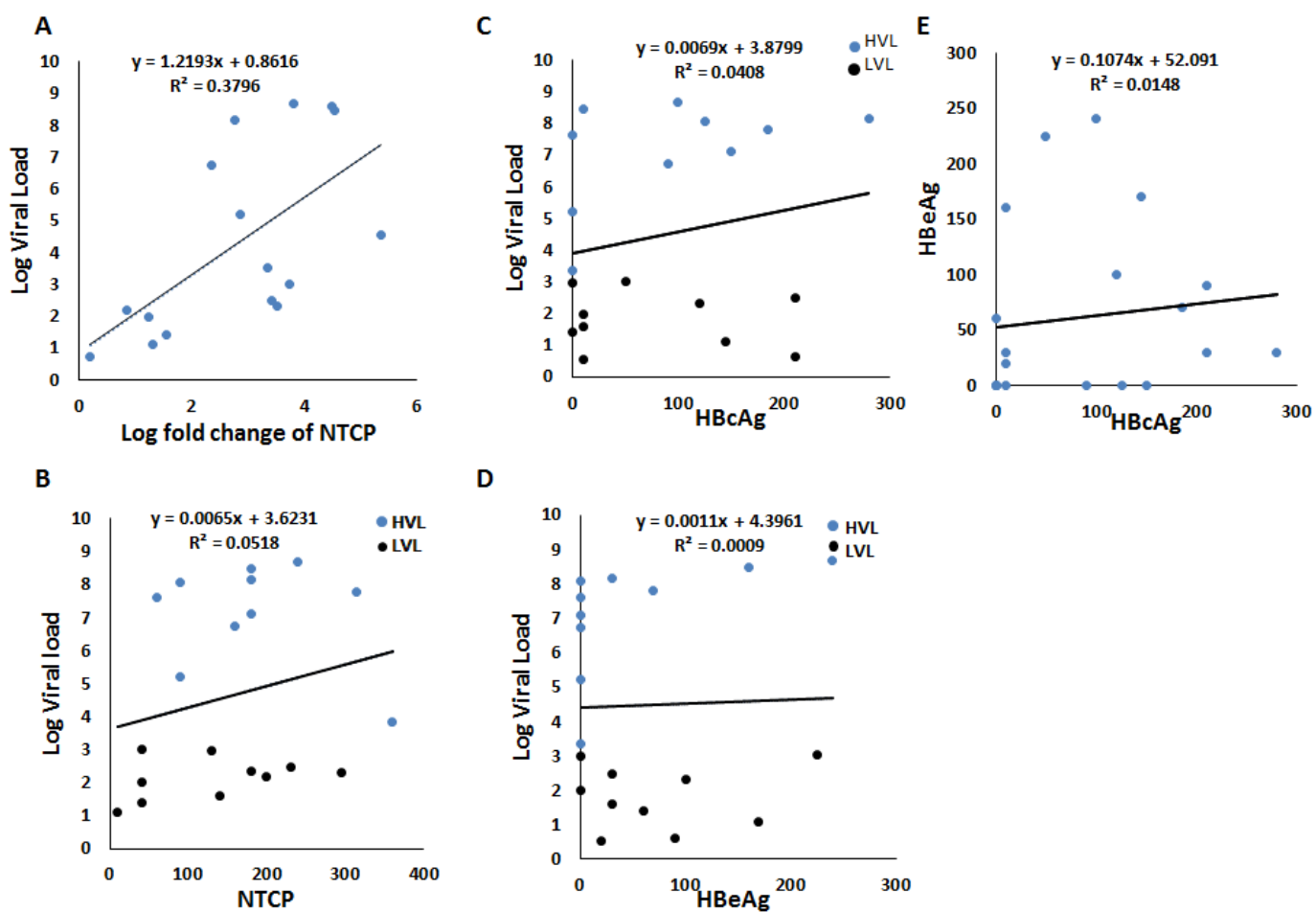

Figure 5 Dot plots showing correlation between log fold change of NTCP expression load $\left(\mathrm{PC}=0.7118, \mathrm{R}^{2}=\right.$ 0.3796, $\left.\mathrm{p}=0.0027 * * ; \mathrm{p}^{* *} 0.0021\right)$ (A) IHC score of NTCP (PC $\left.=0.3577, \mathrm{R}^{2}=0.0518\right)$ (B), IHC score of HBcAg $\left(\mathrm{PC}=0.04907, \mathrm{R}^{2}=0.0139\right)(\mathbf{C}) \mathrm{IHC}$ score of HBeAg $\left(\mathrm{PC}=-0.05213, \mathrm{R}^{2}=0.0009\right)(\mathbf{D})$, with peripheral viral load. Correlation coefficient was calculated using Spearman's correlation test. (E) Correlation of IHC score of HBeAg with IHC score of HBcAg in placental tissues $\left(\mathrm{PC}=0.4015, \mathrm{R}^{2}=0.0112\right.$ ). We further analyze the association between IHC score of $\mathrm{HBeAg}$ and $\mathrm{HBcAg}$ in the placental samples but did not find statistically significant association between them $\left(P C=0.1153, \mathrm{R}^{2}=0.0148\right.$ ) (Figure $5 \mathrm{D}$ and Supplementary Figure 3).

\section{Discussion}

176 As HBV vertical transmission is the primary cause of chronic HBV infection worldwide[12], inhibiting it is the most effective approach towards eradicating chronic HBV infection. Infants infected with HBV face the risk of severe complications due to genetic, epigenetic, and environmental factors. In spite of universal vaccination program, $5 \%$ or more of vaccinated newborns still do not achieve protective levels of anti-hepatitis B virus surface antigen titers [13]. The precise mechanism behind HBV vertical transmission is not known. Our previous study showed presence of ASGPR on placenta and circulating dendritic cells which facilitates the endocytosis of $\mathrm{HBV}^{7}$. Paganelli et al., also found presence of ASGPR on umbilical cord matrix stem cells (UCMS) [14], and showed its competitive inhibition prevents binding and uptake of HBV. In order to establish infection, HBV must bind to its specific receptor. NTCP is a primary receptor of HBV. It was known to present only on hepatocytes and is responsible for the hepatotropism of HBV. It's presence on non-hepatic cells is not well known. Presence of NTCP is critical to make in vitro culture systems susceptible for HBV infection [15]. Indicating that its presence 


\section{Materials and Method:} transmission.

\section{Study Groups:} receiving anti-HBV drugs.

on non-hepatic tissues can make them susceptible towards infection. Here, we far the first time report the presence of NTCP on placenta along with HBV replication markers. HBeAg is a marker of active replication. Previous studies suggested that HBeAg can cross the placenta but it was only based on the serological findings of mother and newborn. In our present study we not only observed the presence of $\mathrm{HBeAg}$ in the placental cells / trophoblasts but also in 50\% cases who were negative for $\mathrm{HBeAg}$ in circulation were positive in placenta along with HBV DNA strongly suggests that placenta can also serve as a host tissue for HBV replication.

We understand that this novel finding provides a mechanistic basis for vertical transmission of HBV and also opens up potential strategies for arresting vertical transmission targeted at NTCP blocking by treatment with Myrcludex B and/or through other alternative strategies may be used for therapeutic intervention of vertical

Our study suffers from the limitation of small sample size and the inability to experimentally demonstrate the prevention of HBV infection of placental tissues through blockage of NTCP receptor.

HBV pregnant females: A total of 27407 pregnant females were screened for HBsAg during their antenatal routine check-up in the in a tertiary care teaching hospital in central India over a period of 2 years from 20202021. A total of 432 of pregnant females were found positive for HBsAg (1.57\%) and negative for hepatitis A, hepatitis $\mathrm{C}$, hepatitis $\mathrm{E}$ and human immunodeficiency virus (HIV). None of the pregnant females had any systemic illness, autoimmune disease or inherited metabolic disorder. Out of 432, we were able to follow $59 \mathrm{HBsAg}+\mathrm{ve}$ pregnant females, till delivery due to loss of follow ups, lack of consent and other complications. Out of 59, 22 had HBV DNA $\geq 2000 I U / m l, 19$ had HBV DNA <2000IU/ml and in 18 samples HBV DNA was below the detection limit. A total $41 \mathrm{HBsAg}+\mathrm{ve}$ and 10 healthy pregnant females were included in this study.

HBsAg Positive mothers ( $\boldsymbol{n}=41)$ : HBsAg positive subjects were divided in two groups on the basis of viral load according to INASL guidelines 2018 [10]. Pregnant females, had HBV DNA load $\geq 2000 \mathrm{IU} / \mathrm{ml}$ were categorized as High viral load (HVL) group $(n=22)$ and pregnant females, who had HBV DNA load <2000IU/ml were categorized as Low viral load (LVL) group $(\mathrm{n}=19)$.

Control Group (n=10): Pregnant females, who were negative for HBsAg and gave birth to healthy newborns, were identified as healthy and were included in the study as controls.

The Informed consent was obtained from each participant before enrolment in the study. The clinical and biochemical assessment of the subjects was done according to the study protocol. None of the participants was

\section{Sampling of serological and virologic studies:}

At term, $=5 \mathrm{ml}$ of peripheral blood sample was collected in EDTA tubes and placental tissues were obtained from maternal decidua (within 6 hours) of delivery. The plasma was separated within an hour of blood collection and immediately stored at $-80^{\circ} \mathrm{C}$ till further use.

\section{Peripheral HBV DNA Quantification}


HBV DNA quantitation was done with $500 \mu \mathrm{l}$ plasma using COBAS Taq Man HBV test with high purity extraction (Roche Diagnostics, USA), as per the manufacturer's protocol. This real-time PCR assay is based on dual labelled hybridization probe targeting the pre-core and core regions. Results were expressed as IU/ml. The lower limit of detection for the assay was $<6 \mathrm{IU} / \mathrm{ml}$.

\section{DNA and RNA Extraction from placental tissues:}

DNA and RNA was extracted from placental tissues frozen in RNA Liv (Himedia). DNA from approximately 20 mg placental tissue was extracted using QIAmp Blood DNA mini kit, Qiagen, Germany using manufacturer's protocol. Approximately $20 \mathrm{mg}$ placental tissue was homogenised using TissueLyser LT (Qiagen) and total RNA was extracted using QIAamp®RNA Blood Mini kit, Qiagen, Germany according to manufacturer's protocol. DNA and RNA was quantified using NanoDrop ${ }^{\mathrm{TM}}$ Spectrophotometer (Thermo Scientific ${ }^{\mathrm{TM}}$ ). High-Capacity cDNA Reverse Transcription Kit (Applied Biosystems, Vilnius, LT) was used for complementary DNA synthesis using $1 \mu \mathrm{g}$ of RNA.

\section{Detection of HBV DNA in placenta:}

qPCR was performed with PowerUp ${ }^{\mathrm{TM}}$ SYBR ${ }^{\mathrm{TM}}$ Green Master Mix kit, (Applied Biosystems, Vilnius, LT) using an BioRad CFX96 Real Time PCR System (BioRad) with primers specific for total HBV DNA targeting X and core ORF (HBV F: GAGGCTGTAGGCATAAATTGGTC, HBV R: AACTCCACAGAAGCTCCAAATTC). Every sample was independently analysed in triplicates.

Detection of HBcAg and HBeAg in placenta by Immunohistochemistry (IHC):

Five-micron thick sections were cut from Formalin Fixed Paraffin Embedded (FFPE) tissue samples and deparaffinized by xylene followed by heat induced antigen retrieval by boiling at $100^{\circ} \mathrm{C}$ for 10 minutes in citrate buffer $(\mathrm{pH}=6)$. Tissue sections were incubated with the following primary antibodies overnight at $4{ }^{\circ} \mathrm{C}: \mathrm{HBcAg}$ (bsm-2000M; Bioss, 1:25), HBeAg (bsm-2022M; Bioss, 1:25). Polyexcel HRP/DAB detection system, PathnSitu (USA) was used for staining using manufacturer's protocol and counterstaining was done with haematoxylin. Images were captured using Nikon DS-Ri2 camera on a Nikon Eclipse Ci microscope.

cDNA prepared from placental RNA was subjected to qPCR using PowerUp ${ }^{\text {TM }}$ SYBR $^{\text {TM }}$ Green Master Mix kit (Applied Biosystems, Vilnius, LT) on BioRad CFX96 Real Time PCR System (BioRad). The specific exon spanning primers of NTCP were designed (NTCP Fwd: CTTCTGCCTCAATGGACGGT; NTCP Rv: GCCACATTGAGGATGGTGGA). qRT-PCR of every sample was done in triplicates to normalize NTCP expression level; GAPDH was used as reference gene. Subsequently, the relative gene expression values were determined using log of $2^{-\Delta \Delta \mathrm{CT}}$ and specificity of product was assured by single melt peak.

\section{Detection of NTCP in placenta by Immunohistochemistry (IHC):}

Five-micron tissue sections were cut, and deparaffinized by xylene followed by heat induced antigen retrieval using boiling at $100^{\circ} \mathrm{c}$ for 10 minutes in Tris buffer (pH-9). Tissues were then incubated with the primary antibody anti-NTCP (BS1985R; Bioss, 1:1000) for 1 hour at room temperature, further staining was done using Polyexcel HRP/DAB detection system PathnSitu (USA) according to manufacturer's protocol and were counterstained with haematoxylin. Healthy Liver section was taken as positive control. Breast, endometrial and myometrial tissues were also stained and analysed. Images were captured using Nikon DS-Ri2 camera on a Nikon Eclipse Ci microscope. 
IHC slides were score by a pathologist using single blinded strategy. Membranous and / or cytoplasmic staining was considered as positive. IHC score was calculated using formula; IHC score = percentage of positive cells * Score of Intensity of the staining. The Score of Intensity followed 1- Weak, 2- Mild, 3- Moderate and 4- Strong for NTCP and HBcAg and 0- Weak, 1- Mild, 2- Moderate and 3- Strong for HBeAg. IHC score was used to calculate statistical significance.

\section{Statistical Analysis}

All statistical tests were performed using GraphPad Prism for Windows version 8.0.1. Participant's demographics are presented as median with range or mean with standard deviation. Categorical variables are presented as proportions while continuous variables are either presented as mean with standard error (SE) or median with range. Continuous variables were tested for normal distribution by Bartlett's test followed by one way ANOVA (nonparametric) test and Tukey's multiple comparison test to find the significance. Correlation was calculated by Spearman's statistics. All statistical comparisons were two tailed and a $\mathrm{p}$ value of $<0.0332$ was considered statistically significant.

2764. Conclusion, this study, first time demonstrating the presence of NTCP on placenta and in search of probable replication, we interestingly found the presence of HBV DNA and $\mathrm{HBeAg}$ in placental cells of $\mathrm{HBeAg}$ negative subjects in circulation. NTCP blocking by treatment with Myrcludex B and/or through other strategy may be used for therapeutic intervention of vertical transmission. Our findings are providing an insight on the probable new mechanism contributing in vertical transmission of the virus and expected to be useful for therapeutic approaches towards its prevention. In summary, this study provides an insight on the probable mechanism contributing to vertical transmission of the virus, which can be clinically exploited in future for its prevention and treatment.

Author Contributions: Conceptualization, project administration and funding acquisition ,Ashish Kumar Vyas; visualization and writing — original draft preparation, Garima Garg, methodology, data curation, and validation, Garima Garg, Meenu MN and Kajal Patel; formal analysis, Sramana Mukhopadhyay; resources, Nitu Mishra, Sumit Kumar Rawat; writing — review and editing, Anirudh K Singh Ritu Khosla, Jitendra Singh and Shashwati Nema ; supervision and investigation, Shashank Purwar and Debasis Biswas;

288 Funding- This research was funded by Department of Science and Technology, INDIA Grant No. DST/INSPIRE/04/2018/000019 and Department of Science and Technology, Science and Engineering Research Board Grant No CRG/2020/003856 to Dr. Ashish Kumar Vyas.

291 Institutional Review Board Statement: The study was conducted in accordance with the Declaration of 292 Helsinki, and approved by the Institutional Human Ethics Committee of All India Medical Sciences (AIIMS), 293 Bhopal, India (Approval No. EF0110; date of approval 26.12.2019 and EF0232; date of approval 19.04.2021

294 Informed Consent Statement: Informed consent was obtained from all subjects involved in the study Acknowledgments: We thank technical staff of Dept. of Pathology, AIIMS, Bhopal for help in IHC work. Nursing Staff of Sultania maternity hospital, Bhopal for participant enrolment. We thank to Ms. Priyal Gupta and Ms. Bijina John Mathew for their intellectual input. 
302 1. Premkumar M, Kumar Chawla Y. Chronic Hepatitis B: Challenges and Successes in India. Clinical liver disease $3032021 ; 18(3): 111-6$.

304 2. Pande C, Sarin SK, Patra S, et al. Prevalence, risk factors and virological profile of chronic hepatitis B virus 305 infection in pregnant women in India. Journal of medical virology 2011; 83(6): 962-7.

306 3. Zanetti AR, Van Damme P, Shouval D. The global impact of vaccination against hepatitis B: a historical overview. 307 Vaccine 2008; 26(49): 6266-73.

308 4. Pande C KA, Patra S, Trivedi SS, Dutta AK, Sarin SK. High maternal hepatitis B virus DNA levels but not 309 HBeAgpositivity predicts perinatal transmission of hepatitis B to thenewborn. . Gastroenterology 2008; 134:A760.

310 5. Joshi SS, Coffin CS. Hepatitis B and Pregnancy: Virologic and Immunologic Characteristics. Hepatology 311 communications 2020; 4(2): 157-71.

312 6. Vyas AK, Negi P, Patra S, et al. Maternal immunity influences vertical transmission of hepatitis B to newborns.

313 Hepatology communications 2019; 3(6): 795-811.

$3147 . \quad$ Vyas AK, Ramakrishna U, Sen B, et al. Placental expression of asialoglycoprotein receptor associated with 315 Hepatitis B virus transmission from mother to child. Liver International 2018; 38(12): 2149-58.

3168 8. Oswald A, Chakraborty A, Ni Y, Wettengel JM, Urban S, Protzer U. Concentration of Na(+)-taurocholate317 cotransporting polypeptide expressed after in vitro-transcribed mRNA transfection determines susceptibility of hepatoma 318 cells for hepatitis B virus. Scientific reports 2021; 11(1): 19799.

319 9. Lucifora G, Martines F, Calabro S, Carroccio G, Brigandi A, de Pasquale R. HBcAg identification in the placental 320 cytotypes of symptom-free HBsAg-carrier mothers: a study with the immunoperoxidase method. American journal of 321 obstetrics and gynecology 1990; 163(1 Pt 1): 235-9.

322 10. Arora A, Singh SP, Kumar A, et al. INASL position statements on prevention, diagnosis and management of 323 hepatitis B virus infection in India: the Andaman statements. Journal of clinical and experimental hepatology 2018; 8(1): 324 58-80.

325 11. Zhang SL, Yue YF, Bai GQ, Shi L, Jiang H. Mechanism of intrauterine infection of hepatitis B virus. World 326 journal of gastroenterology 2004; 10(3): 437-8.

327 12. Vyas A, Jindal A, Hissar S, Ramakrishna G, Trehanpati N. Immune balance in hepatitis B infection: present and 328 future therapies. Scandinavian journal of immunology 2017; 86(1): 4-14.

329 13. Zhang W, Guo Z, Zhang L, et al. Maternal immunization promotes the immune response of neonates towards 330 hepatitis B vaccine. J Viral Hepat 2013; 20(12): 875-81.

331 14. Paganelli M, Dallmeier K, Nyabi O, et al. Differentiated umbilical cord matrix stem cells as a new in vitro model 332 to study early events during hepatitis B virus infection. Hepatology 2013; 57(1): 59-69.

333 15. Otoguro T, Tanaka T, Kasai H, et al. Establishment of a Cell Culture Model Permissive for Infection by Hepatitis 334 B and C Viruses. Hepatology communications 2021; 5(4): 634-49.

\section{Supplementary Figure 1. Gel image showing specific band of HBV DNA qPCR.}

336 Supplementary Figure 2. Expression of NTCP in different nonpathological tissues detected by 337 Immunohistochemistry (IHC) (A) No staining was observed in Breast tissue, weak/ no staining in (B) 338 Endometrium and (C) Myometrium 


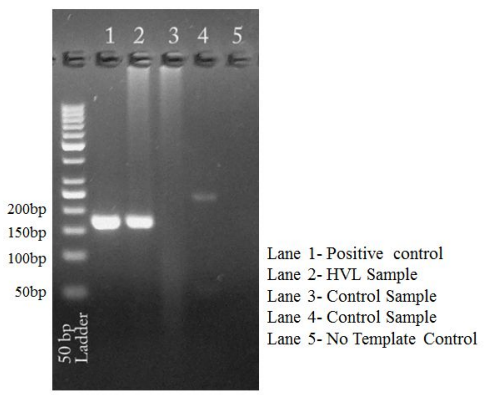




\section{Supplementary Fig 2 Expression of NTCP on other tissues}
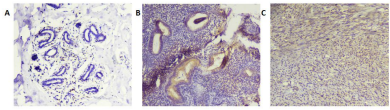


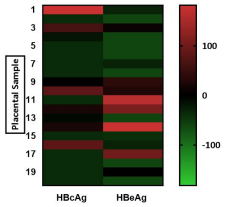

